\title{
EMS Disease Exposure, Transmission, and Prevention: a Review Article
}

\author{
Colton Bitely ${ }^{1} \cdot$ Brian Miller $^{1} \cdot$ Jonathan Glauser ${ }^{1}$ \\ Published online: 11 October 2019 \\ (C) Springer Science+Business Media, LLC, part of Springer Nature 2019
}

\begin{abstract}
Purpose of Review This article aims to review recent literature regarding the risks of disease exposure to pre-hospital providers and the patients they serve, as well as the challenges they face in minimizing transmission and exposure.

Recent Findings Many studies continue to show poor compliance with consistent universal precautions, as well as proper hand hygiene. Vaccination rates are suboptimal despite attempts to encourage compliance. With the spread of multi-drug resistant organisms, new techniques of decontamination need to be investigated.

Summary There remains a general lack of information and studies regarding the risks of disease exposure and transmission to EMS providers despite the significance hazards their profession can pose. However, there remains a continued theme throughout the majority of EMS and pre-hospital studies, demonstrating that hand washing and consistent use of personal protective equipment remains a persistent, preventable means of disease exposure and transmission.
\end{abstract}

Keywords Emergency medical services $\cdot$ Exposure $\cdot$ Transmission $\cdot$ PPE $\cdot$ Contamination $\cdot$ Decontamination $\cdot$ Infectious disease

\section{Introduction}

EMS and pre-hospital providers face special challenges when it comes to disease exposure, which not only puts them at risk, but also poses additional threat to patients under their care. These providers are at several disadvantages when compared with in-hospital providers. Given their initial patient exposure, there is a general lack of information as regards diagnosis and past medical history. They are also at increased risk of exposure with the possibility of insufficient personal protective equipment given certain clinical scenarios. Frequently, hand washing is either limited or altogether unavailable based on circumstances, and thorough cleaning of equipment can be

This article is part of the Topical Collection on Infectious Disease

Jonathan Glauser

jglauser@metrohealth.org

Colton Bitely

cbitely@metrohealth.org

Brian Miller

bmiller1@metrohealth.org

1 Department of Emergency Medicine, MetroHealth Medical System, 2500 MetroHealth Drive, Cleveland, OH 44109, USA challenging, making the providers and equipment potential vectors for disease transmission. In addition, outbreaks of new viruses and resistant pathogens pose new challenges in specific identification and in protecting providers and patients alike. Further complicating matters is the lack of available research and difficulty performing studies given the inherent nature of these limitations.

\section{Needle-Stick Injuries and Vaccinations}

Among healthcare workers, EMS providers are known to have relatively high risk of sustaining needle-stick injuries (NSI). This is logically related to the emergency nature of their work, the mobile nature of their work environment, ambient lighting or space limitations, and the speed at which their procedures need to be done. More recently though, several case studies have been reported which identify a specific medical condition that is uniquely associated with needle sticks: hypoglycemia. An unconscious diabetic patient predisposes the healthcare worker to increased risk of a NSI. The lancets on a glucometer have a very small needle that can be hidden, and sometimes patients do not dispose of these used items properly. The Huber needles on insulin pumps have also been described to have increased risk [1]. 
Hepatitis B, C, and HIV are the most dreaded viral infections, and constitute the most realistic concerns for disease transmission in needle-stick injuries. Familiarity with local disease reporting is crucial to ensuring EMS providers are aware when exposures requiring prophylaxis or additional testing occur. See Fig. 1 for a list of reportable diseases in
Ohio; these can vary from state to state. The universal precautions published by the CDC are not necessarily different for EMS compared with other healthcare workers; however, the compliance can be more challenging. Considering the risk and prevalence of these types of exposures, a protocol for such exposures should be in place in EMS systems as well as health

\section{Know Your ABCs: A Quick Guide to Reportable Infectious Diseases in Ohio From the Ohio Administrative Code Chapter 3701-3; Effective May 1, 2015}

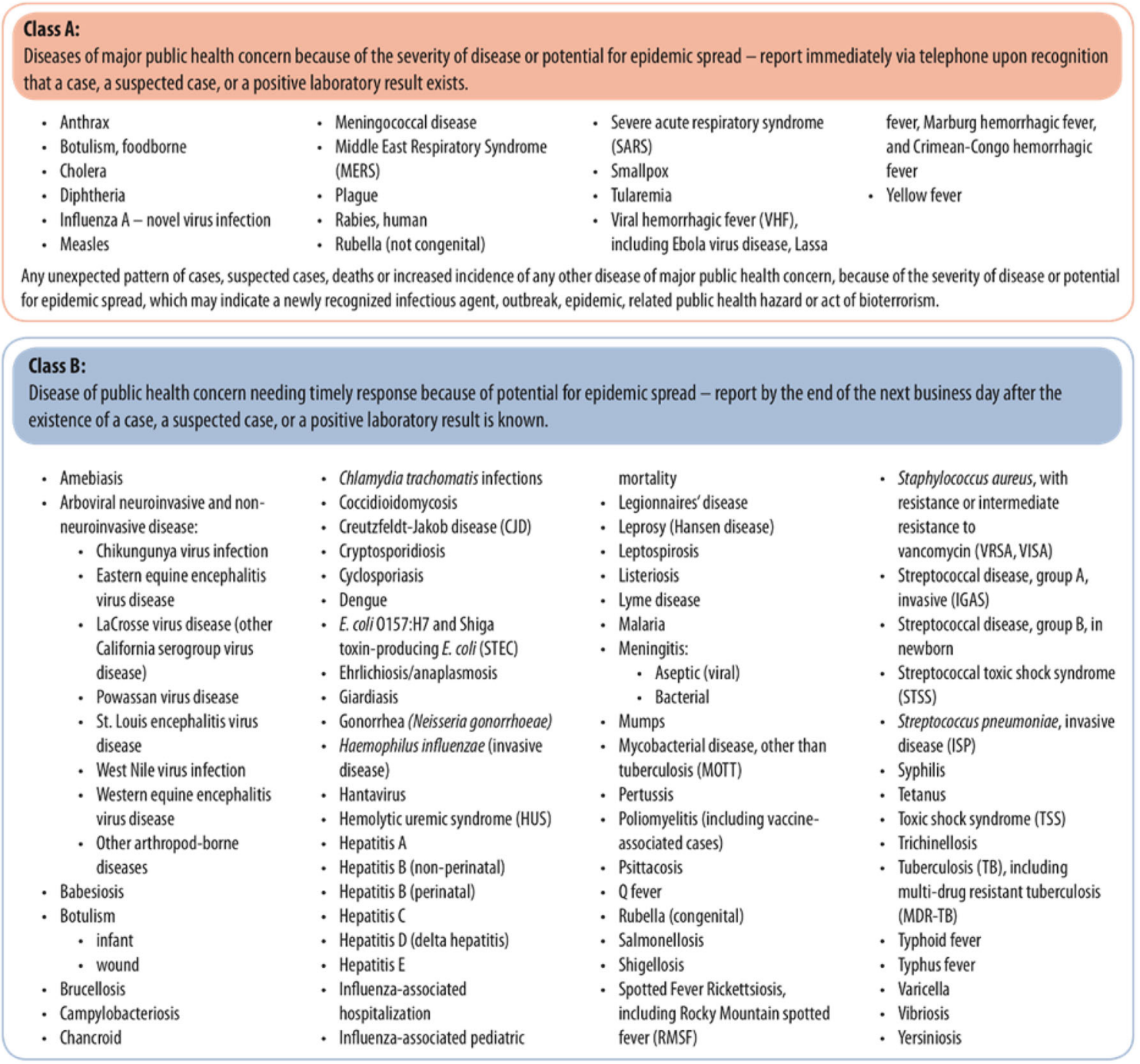

Class C:
Report an outbreak, unusual incident or epidemic of other diseases (e.g. histoplasmosis, pediculosis, scabies, staphylococcal infectic
of the next business day.
$\begin{array}{lll}\text { Outbreaks: } & \text { - Healthcare-associated } & \text {. Waterborne } \\ \text { - Community } & \text { - Institutional } & \text { - Zoonotic } \\ \text { - Foodborne }\end{array}$

NOTE:

Cases of AIDS (acquired immune deficiency syndrome), AIDS-related conditions,

HIV (human immunodeficiency virus) infection, perinatal exposure to HIV,

all CD4 T-lymphocyte counts and all tests used to diagnose HIV must be reported on forms and in a manner prescribed by the Director.

\section{(4) Ohio}

Fig. 1 Reportable diseases and the conditions under which they should be reported for the state of Ohio, USA 
systems. An example of our own institution's protocol regarding HIV testing and response is seen in Fig. 2 (see figure), although screening for other diseases such as hepatitis are included in these protocols. In general, wearing gloves has high compliance, while correct use and disposal of needles has a higher failure rate. Specifically, recapping used needles is a practice that is commonly done despite safety designs incorporated in the needle/syringe system. There is high correlation between certification level and knowledge of the recommended universal precautions: advanced life support (ALS) providers understand and apply the recommendations more consistently than basic life support (BLS) providers. Ironically, the ALS providers report a higher rate of NSI and blood exposures, but are more likely to use needles during their care, and to engage in more intense resuscitation techniques. Forgetfulness, impaired dexterity and vision, and the

\section{NON-EMPLOYEE EXPOSURE \\ QUICK ALGORITHM}

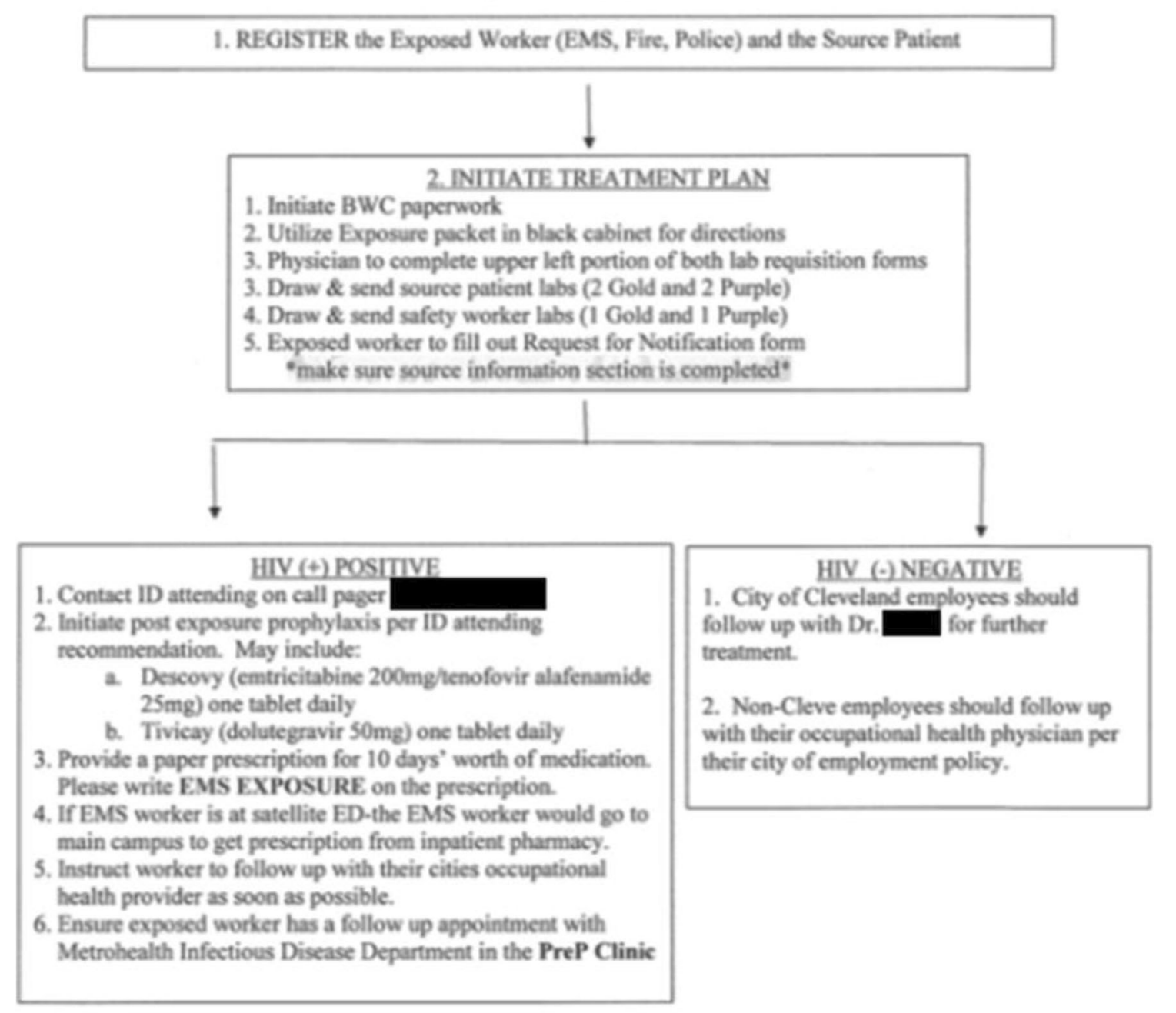

\section{REGARDLESS OF RESULT}

- Fax completed Request for Notification form to Infection Control

- Scan to the preprogrammed Infection Control tab in fax machine.

- Leave a message on Infection Control hotline

Fig. 2 Protocol for EMS workers with disease exposure and process regarding HIV results 
time required for correct application are three reasons cited by EMS workers for non-compliance with universal precautions. Treating a patient who is at "low risk for transmission of disease" was a reason cited for failure to wear gloves, a recommendation that had $83 \%$ compliance in a large survey. Published results of this survey made valuable conclusions in its summary. EMS providers are prone to unintentional NSI due to movement (vehicles and patients), lack of convenient access to sharps containers, and the presence of multiple rescuers. Emphasis on compliance with known universal precautions should be increased among EMS providers [2]. While this likely poses one of the greatest threats to EMS workers, there are not an abundance of updated studies regarding the risk of disease transmission to workers in this setting.

Immunizations in the healthcare setting are an important means of preventing disease burden. It has been shown that despite education and awareness of the risks of disease transmission, healthcare workers, including pre-hospital providers frequently have low compliance rates with vaccines [3]. Identifying the barriers to vaccinations is imperative. Influenza is one area of focus that has potential for significant reduction in transmission and overall prevention with vaccination. Rates of vaccination are consistently low in the general population as well as in health care providers, with many reasons cited for acceptance or refusal [4, 5]. Finding motivators for and identifying obstacles that prevent vaccination, as well as increasing education among providers could be a simple way to help prevent disease transmission both to patients and providers during outbreaks of influenza $\left[6^{\bullet}\right]$.

\section{Hand Hygiene, Universal Precautions, and Decontamination}

EMS providers primarily work in small, enclosed spaces, and these spaces are subject to exposure to many pathogens and bodily fluids that makes them prominent vectors for disease exposure and transmission. A large part of recent research focuses on hygiene and sanitation efforts, as well as the training and education needed to implement it. Efforts are made to keep workspaces clean; however, the demanding and fastpaced nature, lack of time, resources, or even dedicated cleaning time and staff (such as is available in the hospital setting) makes it more difficult to achieve a more sterile environment. This lack of sterility, along with the worsening prevalence of multi-drug-resistant organisms (MDRO), puts both the health care providers and other patients at increased risk. Provider compliance with standard hygiene and use of PPE, as well as proper disinfection techniques can help decrease the disease transmission and exposure risk of such harmful pathogens [7-9]. The increasing prevalence of MDRO also increases the need for pre-hospital provider awareness and knowledge regarding actions needed to combat transmission of not only methicillin-resistant Staphylococcus aureus (MRSA), but also other increasingly common pathogens such as vancomycin-resistant Enterococcus (VRE) and Clostridium difficile. Basic life support (BLS) and advanced life support (ALS) providers alike have been shown to lack education regarding the risks of these pathogens and the steps needed to limit their exposure and transmission. In one study, fewer than half of providers had even heard of some pathogens, and reported that increased awareness would prompt increased compliance with PPE and proper decontamination [10].

Proving and studying the transmission of such pathogens is difficult during actual transport with real patients and actual organisms. However, there have been efforts in attempting to document this, as well as in implementing new cleaning strategies to counteract disease transmission. One such study looked at standard protocol versus an updated protocol using a surrogate bacteriophage to represent bacteria and viruses $[11 \bullet \cdot]$. The authors found that in addition to decreased transmission with updated cleaning protocols, providers' hands were the primary vectors for pathogen transmission. This further illustrates the importance of basic hand hygiene and compliance with PPE. They also found that promotion of decontamination habits and formal training in updated techniques was needed. Another study again emphasized that pre-hospital providers are again major vectors of pathogens [12]. This also reiterated the fact that despite well-known recommendations from the Centers for Disease Control and Prevention, some EMS providers still do not practice appropriate hand washing techniques during patient care, increasing their potential for disease exposure and spread. This was again demonstrated in a study with self-reported compliance with hand hygiene and use of PPE. It, like many studies, showed that even with an understanding of the importance of hand washing and using personal protective equipment properly, there was a distinct lack of compliance among many providers, leaving significant room for improvement [13]. Alternatively, while proper hand hygiene is crucial, the surrounding environment still requires attention [14]. While there remains the constant need to decontaminate between patients as much as possible, improved techniques should be investigated. While decontamination techniques can be improved, it has been suggested that more intense practices and protocols may decrease the risk of persistent contamination in areas where standard cleaning techniques may not be applicable [15]. There is usually a short period of time between the transport of one patient and the next, especially in busy metropolitan and inner-city areas. This, among other complicating factors, is one of the many reasons cited by pre-hospital providers for decreased use of personal protective equipment, which subsequently leads to increased risk of injury or transmission [16]. It has also been shown that even with awareness of the need to disinfect contaminated surfaces, the lack of time, and sometimes 
equipment frequently poses significant challenge to ensuring a clean workspace $[17 \bullet]$.

\section{Ebola and MERS}

EMS providers are faced with transport of many different types of patients. They often have limited history, which may limit their ability to prepare for and protect themselves against dangerous communicable pathogens. Ebola virus and Middle Eastern Respiratory Syndrome are two of the most recent outbreaks of highly communicable diseases that heightened awareness for disease transmission, which warrant mention. Events such as Ebola virus outbreaks, as well as other highly communicable diseases like MERS, raise new concerns and highlight the need for updated protocols, as well as the increased need for education and training and preparation for such situations. This not only applies to the EMS workers, but to the entire emergency response system. This highlights an important need in the emergency medical response system for adaptability, rapid implementation of new protocols, and ability to widely disseminate information to workers to help prevent the transmission of these dangerous, easily transmissible diseases $[18,19]$. Similar to other infectious diseases, the lack of provider compliance, for several reasons, remains a significant issue in disease prevention. The recent MERS outbreak prompted reassessment of reasons for PPE failure. One study revealed multiple reasons, including poor fit, especially for women, anxiety about the donning of PPE, and even low confidence in the PPE efficacy to protect from disease [20]. This is important due to the fact that health care providers are often a significant proportion of infected patients and risk further spread to patients [21]. Proper fitting PPE is especially important in respiratory illnesses like MERS, and providers should undergo proper fitting. It has been shown that there has been poor understanding of the appropriate use of PPE - requiring appropriate use of N95 masks - and isolation of patients in well-established diseases such as tuberculosis [22], so it is imperative to provide adequate information and training for these emerging illnesses and the infection control challenges they pose. While there are similarities between SARS and MERS, the latter has generally shown little human-human transmission. It does, however, have a very high mortality rate and pandemic potential, and travelers pose great risk for the spread of the virus [23], making recognition of suspicious symptom complexes and identification of recent travel a priority for EMS providers.

\section{Conclusion}

Pre-hospital providers and the patients they serve are at heightened risk for disease exposure and transmission. They face unique challenges with regard to prevention of transmission and decontamination of their workspace environment. There is an ever-growing prevalence of multi-drug resistant organisms that further complicates the process, and brings to light the need for investigation of improved cleaning and decontamination protocols. While some of the challenges are unique to the environment in which prehospital personnel practice, there remains an overwhelming abundance of studies revealing that following simple universal precautions, such as proper hand hygiene and improved compliance with the use of personal protective equipment, there remains a great deal of room for improvement. There also remains a considerable amount of need for improved education regarding the various pathogens, the dangers they pose not only to the patients but the providers as well, and the techniques available to help decrease the spread and transmission of aforementioned pathogens.

Acknowledgments The authors would like to thank Dr. Thomas Collins for reviewing their article.

\section{Compliance with Ethical Standards}

Conflict of Interest Colton Bitely, Brian Miller, and Jonathan Glauser declare no conflict of interest.

Human and Animal Rights and Informed Consent This article does not contain any studies with human or animal subjects performed by any of the authors.

\section{References}

Papers of particular interest, published recently, have been highlighted as:

- Of importance

•- Of major importance

1. McGuire-Wolfe C. A previously unidentified risk of needlestick injury in the emergency medical services setting. Am J Infect Control. 2014;42(3):325.

2. Harris S, Nicolai L. Occupational exposures in emergency medical service providers and knowledge of and compliance with universal precautions. Am J Infect Control. 2010;38(2):86-94.

3. Lu P, Euler G. Influenza, hepatitis B, and tetanus vaccination coverage among health care personnel in the United States. Am J Infect Control. 2011;39(6):488-94.

4. Corace K, Prematunge C, McCarthy A, Nair R, Roth V, Hayes T, et al. Predicting influenza vaccination uptake among health care workers: what are the key motivators? Am J Infect Control. 2013;41(8):679-84.

5. Hakim H, Gaur A, McCullers J. Motivating factors for high rates of influenza vaccination among healthcare workers. Vaccine. 2011;29(35):5963-9.

6. Moser A, Mabire C, Hugli O, Dorribo V, Zanetti G, Lazor-Blanchet $\mathrm{C}$, et al. Vaccination against seasonal or pandemic influenza in emergency medical services. Prehosp Disaster Med. 2016;31(2): 
155-62 This study reveals hesitations to vaccinations with some insights to limitations in trying to enforce compliance.

7. Wepler M, Stahl W, von Baum H, Wildermuth S, Dirks B, Georgieff $M$, et al. Prevalence of nosocomial pathogens in German ambulances: the SEKURE study. Emerg Med J. 2015;32(5):409-11.

8. Orellana R, Hoet A, Lu B, Anderson S, Stevenson K. Methicillinresistant Staphylococcus aureus in Ohio EMS providers: a statewide cross-sectional study. Prehosp Emerg Care. 2016;20:184-90.

9. Varona-Barquin A, Ballesteros-Peña S, Lorrio-Palomino S, Ezpeleta G, Zamanillo V, Eraso E, et al. Detection and characterization of surface microbial contamination in emergency ambulances. Am J Infect Control. 2017;45(1):69-71.

10. Liang S, Vantassell P, Crowe R, Froelke B, Marschall J, Bentley M. Knowledge, attitudes, and practices regarding infection prevention among emergency medical services providers. Am J Emerg Med. 2015;33(5):725-7.

11.• Valdez M, Sexton J, Lutz E, Reynolds K. Spread of infectious microbes during emergency medical response. Am J Infect Control. 2015;43(6):606-11 This study is demonstrated EMS providers as significant vectors while also reiterating lack of consistent hygiene practices.

12. Teter J, Millin M, Bissell R. Hand hygiene in emergency medical services. Prehosp Emerg Care. 2014;19(2):313-9.

13. Barr N, Holmes M, Roiko A, Dunn P, Lord B. Self-reported behaviors and perceptions of Australian paramedics in relation to hand hygiene and gloving practices in paramedic-led health care. Am J Infect Control. 2017;45(7):771-8.

14. Noh H, Shin S, Kim N, Ro Y, Oh H, Joo S, et al. Risk stratificationbased surveillance of bacterial contamination in metropolitan ambulances. J Korean Med Sci. 2011;26(1):124-30.

15. Alrazeeni D, Alsuif MS. Nosocomial infections in ambulances and effectiveness of ambulance fumigation techniques in Saudi Arabia. Phase I study. Saudi Med J. 2014;35(11):1354-60.
16. Oh H, Uhm D. Occupational exposure to infection risk and use of personal protective equipment by emergency medical personnel in the Republic of Korea. Am J Infect Control. 2016;44(6):647-51.

17. Barr N, Holmes M, Roiko A, Dunn P, Lord B. Challenges for environmental hygiene practices in Australian paramedic-led health care: a brief report. Am J Infect Control. 2018;46(6):723-5 Showed that despite very frequently working in contaminated conditions, many EMS workers are still under-educated and unprepared on the procedures and methods for decontamination.

18. Isakov A, Miles W, Gibbs S, Lowe J, Jamison A, Swansiger R. Transport and management of patients with confirmed or suspected Ebola virus disease. Ann Emerg Med. 2015;66(3):297-305.

19. Le A, Buehler S, Maniscalco P, Lane P, Rupp L, Ernest E, et al. Determining training and education needs pertaining to highly infectious disease preparedness and response: a gap analysis survey of US emergency medical services practitioners. Am J Infect Control. 2018;46(3):246-52.

20. Kang J, Kim E, Choi J, Hong H, Han S, Choi I, et al. Difficulties in using personal protective equipment: training experiences with the 2015 outbreak of Middle East respiratory syndrome in Korea. Am J Infect Control. 2018;46(2):235-7.

21. Al-Tawfiq J, Perl T. Middle East respiratory syndrome coronavirus in healthcare settings. Curr Opin Infect Dis. 2015;28(4):392-6.

22. Knowledge and adherence to guidelines on the infection control of tuberculosis: survey among health care personnel in South Korean general hospitals. Am J Infect Control. 2008;36(5):E142-3.

23. Sampathkumar P. Middle East respiratory syndrome: what clinicians need to know. Mayo Clin Proc. 2014;89(8):1153-8.

Publisher's Note Springer Nature remains neutral with regard to jurisdictional claims in published maps and institutional affiliations. 